\title{
Panikattacken mit frühem und spätem Beginn: Unterschiedliche pathogenetische Mechanismen?
}

\author{
Early- and Late-Onset Panic Attacks: Evidence for Different Pathogenic Mechanisms?
}

Hans-Ulrich Wittchen, Axel Perkonigg

Max-Planck-Institut für Psychiatrie, Klinisches Institut, Klinische Psychologie, München

\begin{abstract}
Zusammenfassung und Schlüsselwörter
Panikattacken sind mit einer Lebenszeitprävalenz von ungefähr 15\% ein relativ häufiges Phänomen im Gegensatz zu einer vollen Panikstörung, die eine Prävalenz von $2,3-3 \%$ aufweist. In der vorliegenden epidemiologischen Untersuchung $(n=481)$ einer bundesweiten repräsentativen Stichprobe wurde geprüft, ob früh (vor dem 25. Lebensjahr) und spät auftretende Panikattacken sich hinsichtlich Symptomatik, Verlaufs- und Komorbiditätsmustern unterscheiden. Neben einer erhöhten Angstsymptomatik, insbesondere bezüglich respiratorischer Beschwerden und der Angst zu sterben, zeigte sich bei Panikattacken mit spätem Beginn ein erhöhtes Risiko für Multimorbidität. Auch entwickelten sich bei dieser Gruppe komorbide Bedingungen schneller. Dagcgen waren Panikattacken mit frühem Beginn und einem erhöhten Risiko für Agoraphobie sowie phobische Störungen verbunden. Die Ergebnisse werden im Hinblick auf pathogenetische Mechanismen und Implikationen für die Planung therapeutischer Interventionen diskutiert.
\end{abstract}

Ätiologie $\cdot$ Komorbidität $\cdot$ Panikattacken $\cdot$ Panikstörungen $\cdot$ Verlauf

\begin{abstract}
Summary and Key Words
Panic attacks with a lifetime-prevalence of about $15 \%$ are quite frequent phenomena as compared to full-blown panic disorders, which show only a prevalence of about $2.3-3 \%$ in the general population. In our epidemiological study of a general population sample $(n=481)$ we tested whether there are differences in symptomatology, course and comorbidity between early (before the age of 25 years) and late panic attacks. In addition to a generally higher symptom score of attacks, especially with regard to respiratory symptoms and fear of death, a higher risk of multimorbidity was found for panic attacks with late onset. Also in this group, comorbid conditions developed significantly faster. On the other hand, panic attacks with early onset had a higher risk for secondary agoraphobia and phobic disorders. The results are discussed with regard to pathogenetic mechanisms and implications for therapeutic intervention.
\end{abstract}

Comorbidity $\cdot$ Course and outcome $\cdot$ Etiology $\cdot$ Panic attacks $\cdot$ Panic disorder

\section{Einleitung}

In einer Reihe epidemiologischer Studien wurde in den vergangenen Jahren nachgewiesen, daß Panikstörungen relativ seltene, jedoch in der Regel besonders schwere Formen von Angsterkrankungen sind. Je nach diagnostischen Kriterien ergaben sich in epidemiologischen Feldstudien [vgl. zusammenfassend Wittchen und Essau, 1993] Prävalenzraten von 2,3\% [nach DSM-III, American Psychiatric Association (APA) 1980] bis 3,5\% [Eaton et al., im Druck] (nach DSMIII-R, APA 1987). Besonders gravierend ist bei diesem. Störungsbild, daß Panikstörungen in der Regel schnell zu professionellem Hilfesuchverhalten, gravierenden Einschränkungen in psychosozialen Bezügen sowie zu einem erheblichen Ausmaß an Komorbidität führen [Wittchen und Essau, 1989; Margraf und Schneider, 1989]. Besonders häufig sind dabei die Entwicklung weiterer Angststörungen, einer sekundären Depression sowie die Entwicklung von Mißbrauch oder Abhängigkeit von Alkohol und Medikamenten. Wittchen und Essau [1993] beschrieben in ihrer Übersichtsarbeit, daß mehr als zwei Drittel aller Panikstörungen langfristig eine dieser Störungen entwickelten.

Obwohl Panikstörungen eine relativ seltene Form psychischer Störungen darstellen, ist die Panikattacke als das pathogenetisch vermeintlich zentrale Schlüsselsymptom der Panikstörung [Ehlers et al., 1988] ein häufiges Phänomen. In Abhängigkeit von der Definition einer Panikattacke erleben 13-16\% der Gesamtbevölkerung zu irgendeinem Zeitpunkt ihres Lebens zumindest einen plötzlichen und völlig unerwarteten Angstanfall, für den weder körperliche noch externe Auslöser zu identifizieren sind [Wittchen, 1988; Eaton et al., im Druck; Wittchen und Essau, 1991]. Derartige vereinzelte Angstanfälle werden - ebenso wie bei der Panikstörung ungefähr doppelt so häufig von Frauen wie von Männern angegeben. Erstmalige Panikattacken treten nach der Mehrzahl aller Untersuchungen besonders häufig in der Adoleszenz und im frühen Erwachsenenalter auf. Dabei zeigt sich nach den Befunden des Epidemiological Catchment Area Program (ECA) [Eaton und Kessler, 1985; Burke et al., 1990] für Frauen eine unimodale Verteilung mit einem Peak um das 28. Lebensjahr, für Männer hingegen eine bimodale Verteilung mit einem Peak um das 19. und einem weiteren Peak um das 40. Lebensjahr (Abb. 1).

Die Diskrepanz zwischen wenigen Panikstörungen und fast $7 \mathrm{mal}$ häufigeren Panikattacken hat in den vergangenen Jahren, im Zusammenhang mit der Aufklärung der Pathogenese, zu verstärkten Forschungsbemühungen angeregt. Dabei ging es vor allem um die Frage, warum nur wenige 
D1 Hatten Sie schon einmal einen Angstanfall, d.h. wurden Sie ganz plötzlich und unerwartet von einem Gefühl starker Angst oder Beklommenheit überfallen, und zwar in Situationen, in denen die meisten Menschen nicht ängstlich wären?

D2 Solche Angstanfälle treten manchmal auf, wenn man in ernster Gefahr ist oder wenn man im Mittelpunkt der Aufmerksamkeit anderer steht. Trat(en) Ihr(e) Angstanfall/-fälle) auch unabhängig von solchen Situationen auf?

WENN JA, FRAGE VOR DEN PRÜFFRAGEN: Können Sie mir einen dieser Angstanfälle etwas näher beschreiben?

BEISPIEL:

DR.:

PB.:

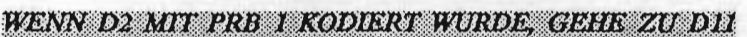

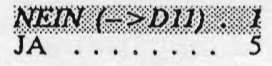

PRB: 12345

D3 SPRACH DER PB. MIT EINEM ARZT DARUBER (D2)?

NEIN $\ldots \ldots 1$

D4 Versuchen Sie jetzt bitte, sich an einen Ihrer schwersten Angstanfälle zurückzuerinnern und an die körperlichen Symptome, die Sie dabei hatten: KODIERE IN SPALTE 1, WIEDERHOLE FALLS NÖTIG: "Hatten Sie während dieses Angstanfalls....!"

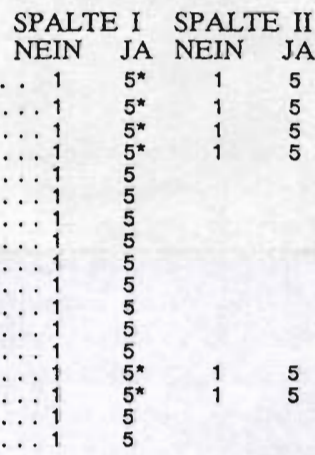

1. Atemnot oder, Schwierigkeiten Luft zu bekommen? ...........

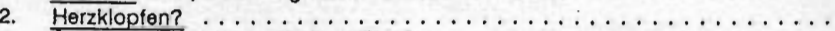

3. Schwindel, Benommenheitsgefühle? ....... Bust oder Magen?

Hatten Sie Kribbel-oder Taubheitsgefühle in den Fingern oder FüBen?

Hatten Sie Erstickungsgefühle?

8. Haben Sie geschwitzt? .............

9. Haben Sie gezittert oder gebebit?

10. Hatten Sie hitzewallungen oder Kälteschauer

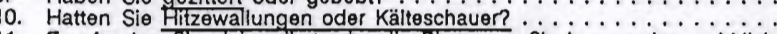

12. Emptanden Sie sich serbst oder die linge um Sle herum als unwirklich?

13. Hatten Sie die Befúrchtung, daß Sie sierben könnten?

14. Verspü Sie die Beturchtung, verruckt zu werden?

15. Hatten Sien Sie einen Brechreiz?

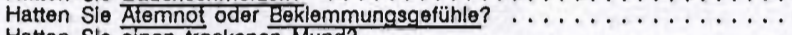

FRAGE FÜR JEDES MIT S* KODIERTE SYMPTOM IN

SPALTE I: Litten Sie unter ... (SX) jemals auch in anderen

Situationen, also wenn Sie keinen Angstanfall hatten?

KODIERE IN SPALTE II

\begin{tabular}{|c|c|c|}
\hline D5: & WURDE WN D4 I IT MAHR ATS HINE $515 *$ KODIERT? & NEIN $(-P D I D, 15$ \\
\hline D6 & $\begin{array}{l}\text { Wann hatten Sie zum (ersten/letzten) Mal einen derartigen } \\
\text { Angstanfall, mit einigen der genannten Symptome wie z.B. } \\
\left(N E N N E \text { EINIGE MIT } 5 / 5^{*} \text { KODIERTEN SYMPTOME AUS }\right. \\
D 4,1-17) ?\end{array}$ & 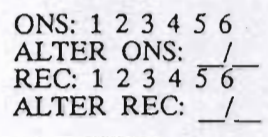 \\
\hline D7 & $\begin{array}{l}\text { Gab es jemals eine Zeitspanne von mehr als einem Monat, in } \\
\text { der Sie jede Woche mindestens } 4 \text { solcher Angstanfälle batten? }\end{array}$ & $\mathrm{NEIN}, D(0) \cdots \cdot 1$ \\
\hline D8 & $\begin{array}{l}\text { Hatten Sie jemals } 4 \text { Angstanfälle innerbalb von } 4 \text { aufein- } \\
\text { anderfolgenden Wochen? }\end{array}$ & $\mathrm{NEIN}, \rightarrow D T 0) \quad \frac{1}{5}$ \\
\hline D9 & $\begin{array}{l}\text { Hatten Sie nach einem solchen Angstanfall wochenlang ( } 4 \\
\text { Wochen) ständig Angst davor, wieder einen Angstanfall zu } \\
\text { bekommen? }\end{array}$ & $\begin{array}{l}\text { NEIN } \ldots \ldots \\
\text { JA } \ldots \ldots\end{array}$ \\
\hline D10 & $\begin{array}{l}\text { Traten einige der Symptome, wie z.B. (NENNE BIS ZU } 4 \\
D E R \text { MIT S/5* KODIERTEN SYMPTOME AUS D4 1 - 17) } \\
\text { bei (diesem/einem dieser) Angstanfall/-anfälle) sehr plötzlich } \\
\text { auf und verschlimmerten sich dann innerhalb von wenigen } \\
\text { Minuten? }\end{array}$ & $\begin{array}{l}\text { NEIN } \ldots \\
\text { JA } \ldots\end{array}$ \\
\hline
\end{tabular}

Abb. 2. Interviewfragen zur Ermittlung der Panikattacken und Panikstörungen [Auszug aus dem CIDI, Wittchen und Semler, 1990]. 
wiesen einen Mißbrauch oder eine Abhängigkeit von Alkohol oder anderen Drogen oder Medikamenten auf. Die 6-Monate-Querschnittsprävalenz lag mit 6,9\% für affektive Störungen und 8,1\% für Angststörungen deutlich niedriger.

Unabhängig vom Vorliegen einer dieser DSM-III-Diagnosen wiesen 67 von 483 auswertbaren Personen zumindest einmal im Verlauf ihres Lebens eine Panikattacke gemäß den Kriterien des DIS nach DSMIII auf (Tab. 1).

Auf der Grundlage der Befunde zum ersten Auftreten einer Panikattacke [Burke et al., 1990] wählten wir als Cut-off für die Einteilung der Untersuchungsgruppen für die vorliegende Studie ein Alter von 25 Jahren. Personen mit dem ersten Auftreten einer Panikattacke bis zum 25. Lebensjahr wurden der Gruppe mit frühem Beginn und Personen mit der ersten Panikattacke nach dem 25. Lebensalter der Gruppe mit spätem Beginn zugeordnet. Aufgrund dieser Einteilung lassen sich 18 Fälle unserer Stichprobe der Gruppe mit frühem Beginn und 46 Personen der Gruppe mit spätem Beginn zuordnen. Wie aus Tabelle 1 ersichtlich ist, unterscheiden sich beide Gruppen in keiner der untersuchten soziodemographischen Variablen signifikant. Leicht erhöht erscheint das Alter der Panikattacken mit spätem Beginn, jedoch ließ sich dieser Unterschied nicht statistisch sichern. Somit kann von einer guten Vergleichbarkeit der Gruppen von Panikattacken mit frühem und mit spätem Beginn ausgegangen werden. In der letzten Spalte der Tabelle 1 ist ferner angegeben, welche Merkmale die Probanden mit einer manifesten Panikstörung aufweisen. Auch hier zeigte sich kein signifikanter Unterschied zu den beiden Gruppen mit Panikattacken.

\section{Erhebungsinstrumente}

Die diagnostische Einordnung der Probanden wurde auf der Grundlage der deutschen Version des Diagnostic Interview Schedule (DIS, Version 2) [Wittchen und Rupp, 1981] vorgenommen. Dieses vollstandardisierte diagnostische Interview erlaubt eine computerisierte Diagnosenstellung nach DSM-III für Angst, Somatisierungs-, affektive sowie Abhängigkeitsstörungen, und zwar sowohl für die gesamte Lebensspanne (Lifetime) als auch für die verschiedenen Ebenen des Querschnittsbefundes (4 Wochen, 6 Monate, $1 \mathrm{Jahr}$ ). Die Panikstörung und die Panikattacken wurden mit den in Abbildung 2 dargestellten Fragen aus dem Untersuchungsinstrument DIS ermittelt.

Zusätzlich werden in diesem Interview Daten über das Alter bei Beginn von Kernsymptomen und Störungen erhoben sowie Daten über das erste und das letzte Auftreten und für ausgewählte Bereiche auch Informationen über Verlauf und Häufigkeit von Phasen. Zusätzlich zu der standardisierten diagnostischen Erhebung wurden alle auffälligen Probanden von einem klinisch erfahrenen Psychiater auf der Grundlage eines strukturierten Befragungsbogens bezüglich des Störungsverlaufs wowie spezifischer psychopathologischer Verlaufsaspekte beurteilt.

\section{Auswertung}

Neben einer deskriptiv-statistischen Auswertung wurde das Komorbiditätsrisiko, d. h. die Assoziation von mehr als einer psychopathologischen Kategorie innerhalb einer Person, mit dem sogenannten Odds ratio ausgewertet. Das Odds ratio [Lemeshow et al., 1990] gibt die Stärke der Assoziation zweier Störungen unter Berücksichtigung der Basisraten der jeweiligen diagnostischen Kategorie an.

\section{Ergebnisse}

Unterscheiden sich die Panik-Symptomprofile von Probanden mit frühem und spätem Beginn?

Der Symptomprofilvergleich der 67 Fälle mit frühem und spätem Beginn einer ersten Panikattacke ergibt für die

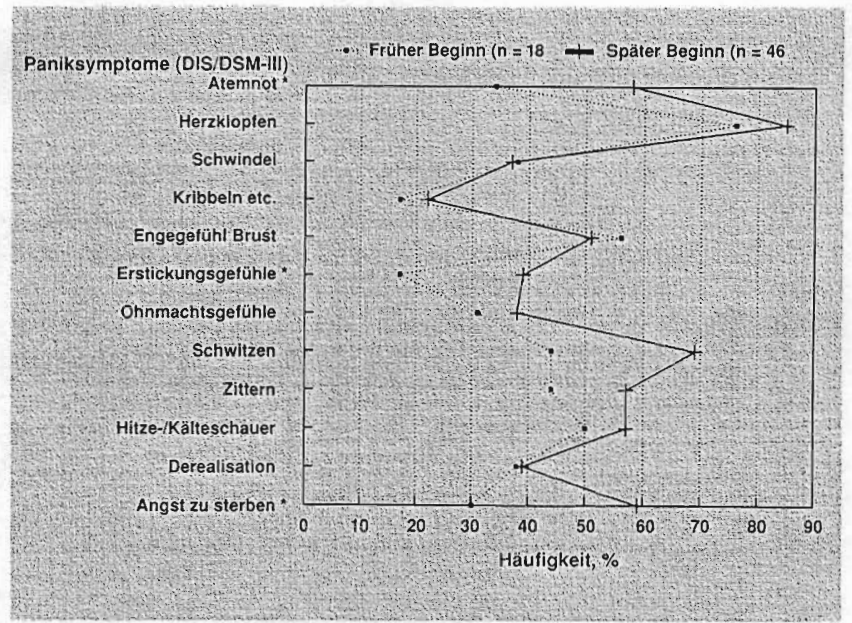

Abb. 3. Panikattacken mit frühem und spätem Beginn: Symptomprofilvergleich. *t-Test (signifikant auf dem 5\%-Niveau).

Gruppe mit späterem Beginn - mit einer Ausnahme (Engegefühl in der Brust) - häufigere panikbezogene Symptome (Abb. 3). Gleich häufig werden Schwindelgefühle, Kribbeln und Taubheitsgefühle in den Extremitäten und Derealisations- und Depersonalisationsgefühle angegeben. Deutlich häufiger weist die Gruppe mit späterem Beginn während des Angstanfalls die Symptome Atemnot, Erstickungsgefühle und «die Angst zu sterben» auf (alle signifikant, $p<0,05$, $\mathrm{t}$-Test). Im Mittel weisen Fälle mit spätem Beginn signifikant $(\mathrm{p}<0,05, \mathrm{t}-$ Test) mehr Paniksymptome auf [Mittelwert (MW) $6,0]$ als Fälle mit frühem Beginn (MW 4,5). Da die beiden Gruppen mit frühem und spätem Beginn sich nicht bezüglich mittlerem Alter und Geschlechtszusammensetzung unterscheiden, kann die erhöhte Angstsymptomatik später Panikattacken nicht auf Stichprobenunterschiede zurückgeführt werden.

\section{Verlauf und Komorbidität}

Tabelle 2 faßt zusammen, welche psychopathologischen Phänomene im weiteren Verlauf nach der ersten Panikattacke beobachtet wurden. Dabei wird deutlich, daß Panikattacken in der überwiegenden Mehrzahl aller Fälle mit einem erheblichen Risiko verbunden sind, nicht nur Panikstörungen oder Agoraphobie, sondern fast ähnlich häufig auch andere psychische Störungen zu entwickeln.

Unabhängig davon, ob wir Fälle mit frühem oder spätem Beginn betrachten, zeigen nur ein Drittel aller Probanden ( $33 \%$ der Fälle mit frühem, 31\% der mit spätem Beginn) im weiteren Verlauf ihres Lebens keine psychopathologischen Komplikationen. Ein Drittel remittieren also offensichtlich spontan. Lediglich 2 Probanden gaben an, eine zweite bzw. dritte Panikattacke, in beiden Fällen Jahre nach der ersten, erlebt zu haben, ohne jedoch das Vollbild einer Panikstörung zu entwickeln.

Die überwiegende Mehrzahl aller Fälle entwickelten eine oder mehrere weitere psychische Störungen. Tabelle 2 gibt im oberen Teil an, wie viele Fälle nur Angststörungen, eine nur depressive Störung oder nur Substanzmittelmißbrauch entwickelten. Im unteren Teil wird gesondert der Prozentsatz für Multi-Komorbidität angegeben, d.h. wie viele Personen 
Tab. 2. Panikattacken mit frühem und spätem Beginn: Komobiditätsmuster und Odds ratios

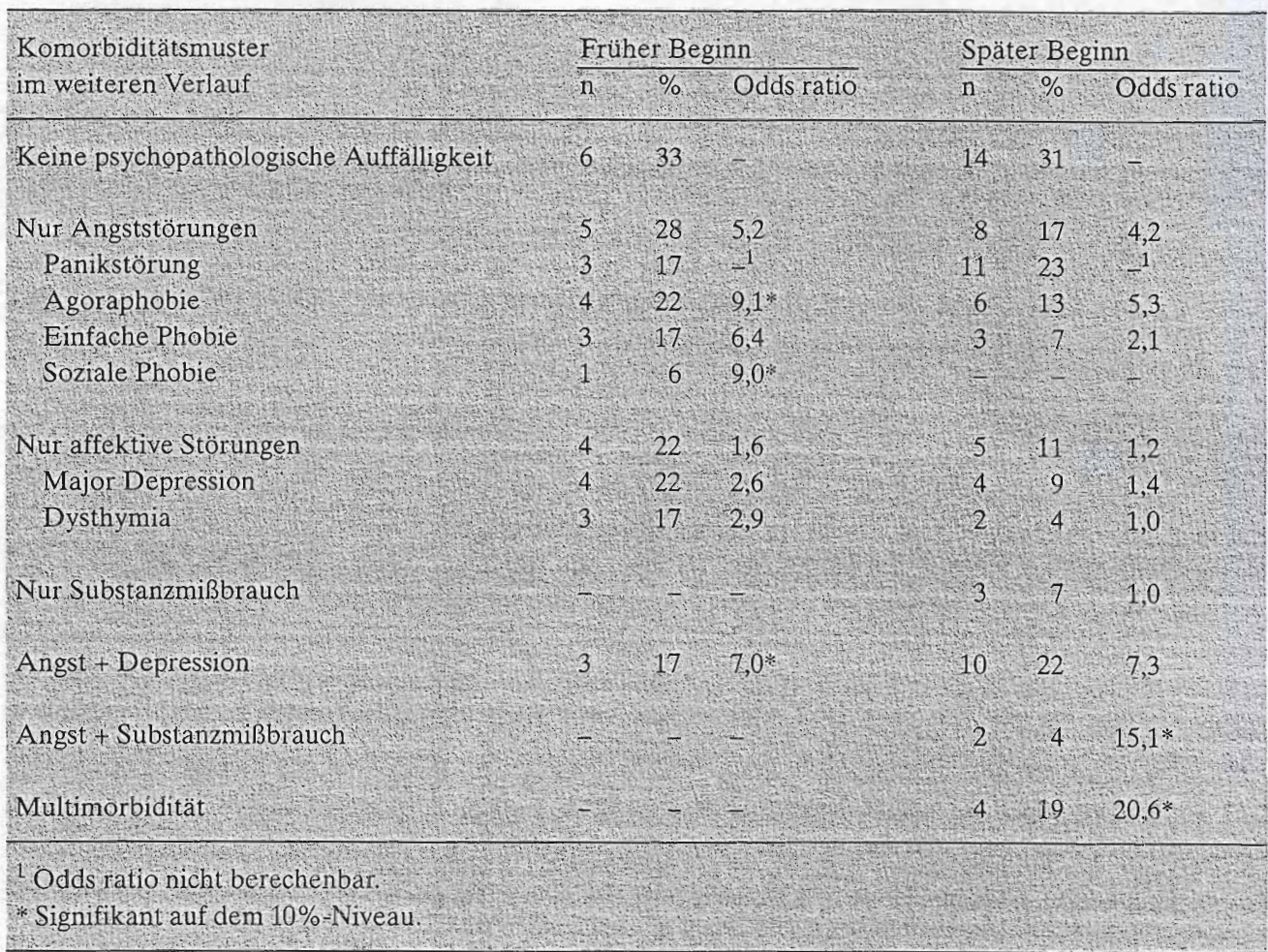

sowohl Angst- als auch depressive Störungen entwickelten, bzw. Angst und Depression und Angststörungen, bzw. depressive und Substanzstörungen. Zusätzlich wird das Odds ratio angegeben, das erkennen läßt, wie hoch das Risiko für frühe oder späte Panikattacken ist, die jeweilige Komorbiditätsbedingung zu entwickeln. Dieses Maß berücksichtigt die jeweiligen Basiswahrscheinlichkeiten für die jeweilige Komorbiditätsbedingung. Aufgrund der kleinen Fallzahlen sind die Konfidenzintervalle groß, so daß nur wenige der Odds ratios auch auf dem 5\%-Niveau zu sichern sind.

Das Risiko, nach einer initialen Panikattacke das Vollbild einer DSM-III-Panikstörung zu entwickeln, ist für beide Gruppen annähernd gleich (frühe $17 \%$, späte $23 \%$ ). Initiale Panikattacken sind ferner kein signifikanter Risikofaktor für rein depressive Störungen, weder für eine Major Depression noch für eine Dysthymie. Zwar deutet hier das Odds ratio von 2,9 an, daß die Wahrscheinlichkeit, eine Dysthymie zu entwickeln, um den Faktor 2,9 erhöht ist, jedoch ist dieser Befund selbst auf dem 10\%-Niveau statistisch nicht signifikant. Übereinstimmend ist ferner in beiden Gruppen das Risiko, erhöht irgendeine Angst und depressive Störung sowie irgendeine Angst und Substanzmißbrauch zu entwickeln. Fälle mit einer frühen Panikattacke haben ein 7 fach erhöhtes Risiko gegenüber Personen, die noch nie eine Panikattacke erlebt haben, im Verlaufe ihres Lebens eine Angststörung und eine Depression zu entwickeln. Es ist bedeutsam, darauf hinzuweisen, daß Panikanfälle an sich in beiden Gruppen allein keinen Risikofaktor für Substanzmißbrauch darstellen. Deutliche Unterschiede ergeben sich jedoch zwischen den beiden Gruppen bezüglich der Assoziationen mit einer Agoraphobie, einer einfachen sowie sozialen Phobie. Während primäre frühe Panikattacken ein extrem hohes Risiko aufweisen, eine Agoraphobie (9,1fach erhöht), eine einfache
Phobie (6,4fach erhöht) sowie eine soziale Phobie (9fach erhöht) zu entwickeln, findet sich in der Gruppe mit spätem Beginn keine signifikante Assoziation; lediglich die Assoziation mit der Agoraphobie (5,3fach) erscheint erhöht. Ein weiterer gravierender Unterschied ergibt sich hinsichtlich des Risikos für Multimorbidität, d.h. der Wahrscheinlichkeit, Angststörungen und Substanzmißbrauch sowie Angststörungen, depressive Störungen und Substanzmißbrauch zu entwickeln. Fälle mit spätem Krankheitsbeginn weisen hier ein 20,6fach erhöhtes Risiko auf.

Zusammenfassend können wir also folgern, daß frühe Panikattacken eher mit der Entwicklung von monosymptomatischer Agoraphobie, sozialer und einfacher Phobie, aber seltener mit einer der Angststörung folgenden «sekundären» Depression assoziiert sind. Späte Attacken hingegen sind mit einem gravierenden Risiko an Multimorbidität, insbesondere von Agoraphobie, Panikstörung, Depression und Substanzmißbrauch, verbunden.

Zeitabstand von Panikattacken und komorbiden Bedingungen Nachdem recht unterschiedliche, sich zeitlich sekundär entwickelnde Komorbiditätsmuster bei Panikattacken mit frühem und spätem Beginn nachgewiesen wurden, haben wir geprüft, inwieweit sich auch unterschiedliche Zeitabstände zwischen initialen Panikattacken und sekundären psychopathologischen Komplikationen finden lassen. Hierzu haben wir aufgrund der retrospektiven Angaben analysiert, wieviel Monate zwischen der ersten Panikattacke und der Entwicklung einer komorbiden Bedingung lagen. Im Falle von Multimorbidität wurde die früheste der verschiedenen komorbiden Bedingungen gewählt.

Abbildung 4 zeigt für die zwei Gruppen mit frühem und spätem Beginn deutliche Unterschiede. Auffallend ist, daß 


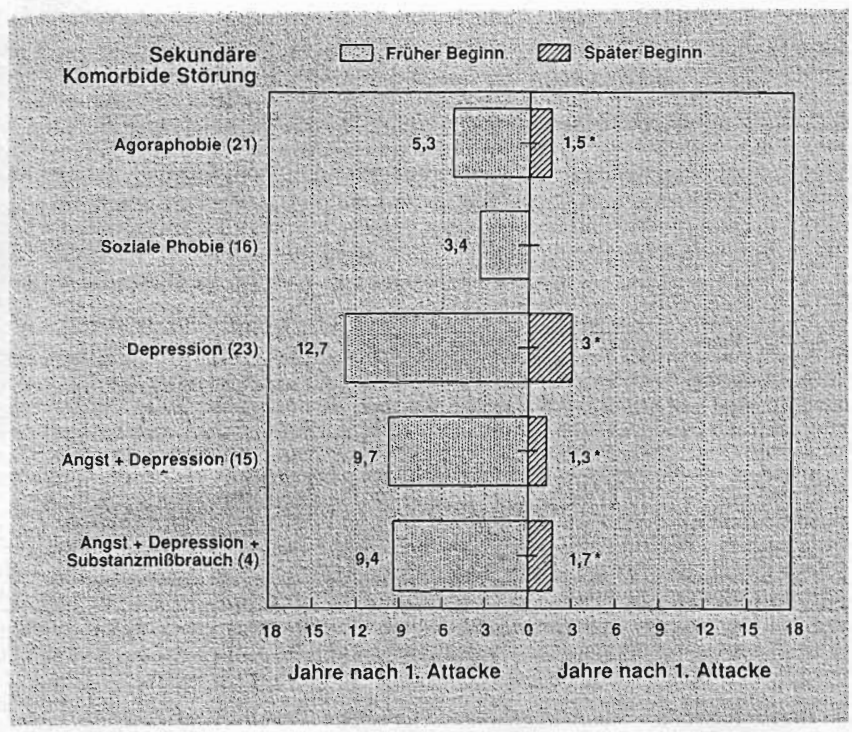

Abb. 4. Zeitraum zwischen der ersten Panikattacke und der Entwicklung einer Komorbidität. Median-Test: *5\%-Niveau.

die Gruppe mit spätem Beginn hochsignifikant schneller komorbide Bedingungen entwickelte. In der Gruppe mit frühem Beginn vergehen im Mittel 5,3 Jahre nach der ersten Attacke, bevor sich eine Agoraphobie, 3,4 Jahre bevor sich eine soziale Phobie und 12,7. Jahre bevor sich eine Major Depression oder eine Dysthymie entwickelt. Die entsprechenden Zeitabstände für die Gruppe mit spätem Beginn sind signifikant ( $p<0,05$; $t$-Test), zum Teil um ein Vielfaches kürzer.

\section{Diskussion}

Vor einer Diskussion dieser Befunde muß auf einige Begrenzungen der vorliegenden Studie hingewiesen werden:

1. Die vorliegende Studie ermöglichte aufgrund der kleinen Fallzahlen keine multivariate Analyse, in der wir insbesondere dem interessanten geschlechtsspezifischen Unterschied, der sich in der Alterskurve andeutet, nachgehen könnten. Deshalb müssen auch die angedeuteten Unterschiede im Symptomprofil-Verlauf mit Vorsicht interpretiert werden. Detailliertere Analysen an einem größeren Datensatz könnten hier möglicherweise deutlichere geschlechtsspezifische Unterschiede aufzeigen.

2. Es sollte berücksichtigt werden, daß die Analysen zum Zeitabstand zwischen der ersten Panikattacke und einer sich entwickelnden morbiden Bedingung aufgrund retrospektiver Angaben der Person zustande kommen. Diese wurden zwar im Rahmen der klinischen Nachuntersuchung bei der Verlaufsdokumentation durch einen Kliniker spezifiziert; die so gewonnenen retrospektiven Daten sollten jedoch aufgrund der zum Teil langen Zeitspanne zwischen initialer Panikattacke und den Komorbiditätsbedingungen mit Vorsicht interpretiert werden.

Unabhängig von diesen Einschränkungen können wir jedoch festhalten: Panikattacken mit frühem und spätem Beginn unterscheiden sich zum Teil deutlich in ihrer Symptomatik. Bei späten Panikattacken treten insgesamt mehr Symptome auf, wobei der Schwerpunkt vor allem auf einer größeren
Häufigkeit respiratorischer Symptome liegt sowie der Angst zu sterben. Inwieweit dieser Symptomprofil-Unterschied durch die häufigere Entwicklung komorbider psychopathologischer Bedingungen erklärt werden kann, muß offen bleiben. Da die Zahl reiner Panikstörungen mit spätem Beginn zu klein für eine differenziertere Analyse ist, kann diese Frage anhand des vorliegenden Datenmaterials nicht eindeutig entschieden werden. Wir können ferner spekulieren, daß bei einer geschlechtsspezifischen Differenzierung Paniksymptome bei Männern mit späterem Beginn noch deutlicher ausgeprägt sind als in der dargestellten Gesamtgruppe. In einer kürzlich durchgeführten Reanalyse der ECA-StudieDaten konnten Eaton und Mitarbeiter [pers. Mitteilung] zeigen, daß Männer mit späterem erstem Auftreten von Panikattacken sehr viel häufiger kardiovaskuläre Symptome, insbesondere Enge und Schmerzen in der Brust, angeben als Personen mit früherem Onset. Inwieweit dieser Befund aufgrund altersspezifischer unterschiedlicher kognitiver Schemata («ältere Fälle konnten psychophysiologische Symptome sensibler wahrnehmen und mit hoher Wahrscheinlichkeit als bedrohlich werten») zustande kommt oder möglicherweise als Hinweis auf tatsächlich vorhandene objektivierbare kardiovaskuläre Symptome zu sehen ist, ist derzeit noch eine offene Frage. Aufgrund der differenzierten Erfassung im psychopathologischen Erfassungsinstrument, dem DIS, konnten zumindest zum Untersuchungszeitpunkt bei keinem der Fälle mit Panikattacken Hinweise auf manifeste körperliche internistische Erkrankungen gefunden werden. Da alle Probanden mit späten Panikstörungen regelmäßig ärztliche und fachärztliche Besuche aufwiesen [Wittchen und von Zerssen, 1987], läßt sich zumindest zum derzeitigen Zeitpunkt dafür keine Evidenz finden.

Deutliche Hinweise fanden wir auf unterschiedliche pathogenetische Mechanismen im weiteren Verlauf. Panikattacken mit einem frühen Beginn scheinen in erster Linie mit der Entwicklung von monosymptomatischer Angstsymptomatik verbunden zu sein. Hier findet sich auch eine deutliche Assoziation bezüglich der in der ätiologischen Literatur häufig betonten pathogenetischen Bedeutung einer Panikattacke. Das Risiko, bei einer frühen Panikattacke eine Agoraphobie zu entwickeln, war gegenüber der Referenzgruppe 9 fach erhöht. In der Gruppe mit spätem Beginn betrug das Risiko lediglich 5,1. Allerdings ist auffällig, daß Panikattacken bei frühem Panikbeginn nicht nur mit der Entwicklung einer Agoraphobie, sondern auch mit einfachen und sozialen Phobien verbunden waren. Insofern ist naheliegend, daß früh auftretende Panikattacken möglicherweise ein relevanter Risikofaktor für alle phobischen Störungen und nicht nur für Agoraphobie und Panikstörung darstellen. Relevant ist in diesem Zusammenhang auch der Befund, daß Panikattacken an sich kein erhöhtes Risiko für depressive Erkrankungen darstellen. Erst wenn manifeste Angststörungen anderer Art vorliegen, kommt es nach unserer Analyse zu einer Entwicklung von Depression. Das Risiko, sowohl Angststörungen wie auch depressive Erkrankungen, die ausschließlich sekundär nach der Angstsymptomatik auftreten, zu entwickeln, war sowohl bei frühen wie auch bei späten Panikattacken 7 fach gegenüber der Referenzgruppe erhöht.

Panikattacken mit späterem Beginn sind sehr viel häufiger als solche mit frühem Beginn mit der Entwicklung von Multi- 
morbidität verbunden, während die Assoziationen zu einzelnen Angststörungen entweder gar nicht oder nur in einem wesentliche geringeren Ausmaß als bei der Gruppe mit frühem Beginn nachzuweisen waren. Das heißt, daß bei Panikattacken mit spätem Beginn sehr viel häufiger Angstkomorbidität im Sinne gleichzeitiger depressiver Störungen sowie Substanzmißbrauch in der weiteren Entwicklung zu erwarten sind. Dieser Befund könnte andeuten, daß bei späten Panikattacken über das veränderte altersspezifische Hilfesuchverhalten unterschiedliche psychogenetische Mechanismen zum Tragen kommen. Aus unseren Analysen in der Münchner Follow-up-Studie [Wittchen und von Zerssen, 1987] wissen wir, daß die Häufigkeit, mit der ältere Probanden ärztliche und fachärztliche Behandlung aufsuchen, 2- bis 3fach höher liegt als bei Probanden in jüngeren Altersgruppen. Hier scheint offensichtlich eine spät auftretende erstmalige Panikattacke häufiger professionelles Hilfesuchverhalten und möglicherweise auch häufiger entsprechende pharmakologische und medikamentöse Therapiestrategien zu triggern, die dann schneller zur Ausbildung eines massiveren Krankheitsverhaltens mit Rückzug und Substanzmißbrauch führen können. Für diese Hypothese spricht auch unser Befund, daß Panikattacken mit früherem und späterem Beginn sich deutlich in bezug auf die Schnelligkeit unterscheiden, in der komorbide psychopathologische Komplikationen auftreten. Während sekundäre Komplikationen in der Gruppe mit frühem Beginn oft erst nach vielen Jahren auftraten, war bei der Gruppe mit spätem Beginn zumeist bereits nach Monaten das volle Komorbiditätsmuster nachweisbar. Besonders schnell trat dies im Zusammenhang mit der Multimorbidität auf, nämlich bei den Fällen, die sowohl Angst wie auch depressive Störungen und Substanzmißbrauch entwickelten.

Diese Befunde geben einen ersten Hinweis darauf, daß es sinnvoll sein könnte, zwischen Panikattacken mit frühem und spätem Beginn zu unterscheiden. Diese Unterscheidung könnte bedeutsame Implikationen nicht nur für die ätiologische Forschung, sondern auch für die Planung therapeutischer Interventionen besitzen. Sollte sich z. B. in unseren laufenden differenzierteren Untersuchungen $\mathrm{zu}$ initialen Reaktionen auf Panikattacken zeigen, daß tatsächlich das altersspezifische Hilfesuchverhalten für die Entwicklung der schnelleren und gravierenden Komorbidität verantwortlich gemacht werden kann, dann sollte die Planung primärärztlicher therapeutischer und insbesondere verhaltenstherapeutischer Interventionen speziell diesem Aspekt Rechnung tragen. Gleichzeitig zeigen unsere Studien auch, daß Panikattacken an sich ein bedeutsamer allgemeiner Risikofaktor für die spätere Entwicklung von anderen Formen psychischer Störungen sein können. Der Umstand, daß zwei Drittel aller Personen mit zumindest einer Panikattacke später massivere psychopathologische Komplikationen entwickeln, unterstreicht die Notwendigkeit, diesem Befund differenzierter nachgehen. In einer derartigen laufenden Reanalyse anderer großer epidemiologischer Studien im Rahmen des Mac Arthur Foundation (Network I, Comorbidity and Boundaries of Affective Disorder) sowie des National Comorbidity Survey [Kessler et al., in press] versuchen wir derzeit, die angedeuteten Hypothesen zu verifizieren.

\section{Literatur}

American Psychiatric Association: Diagnostic and Statistical Manual of Mental Disorders; 3rd ed. Washington, DC, APA, 1980. Deutsche Bearbeitung: Koehler K, Sap H: Diagnostisches und Statistisches Manual Psychischer Störungen. DSM-III. Weinheim, Beltz, 1984. American Psychiatric Association: Diagnostic and Statistical Manual of Mental Disorders; 3rd ed., revised. Washington, DC, APA, 1987. Deutsche Bearbeitung: Wittchen H-U, Saß H, Zaudig M, Koehler K: Diagnostisches und Statistisches Manual Psychischer Störungen. DSM-III-R. Revision. Weinheim, Beltz, 1989.

Burke KC, Burke JD, Regier DA, Rae DS: Age at onset of selected mental disorders in five community populations. Arch Gen Psychiatry 1990;47:511-518.

Eaton WW, Kessler LG (eds): Epidemiologic Field Methods in Psychiatry. The NIMH Epidemiologic Catchment Area Program. Orlando, Academic Press, 1985.

Eaton WW, Kessler RC; Wittchen H-U, Magee WJ: Panic and panic disorders in the United States. Am J Psychiatry (im Druck).

Ehlers A, Margraf J, Roth W: Selective information processing, interoception, and panic attacks, in Hand I, Wittchen $H$-U (eds): Panic and Phobias. 2. Treatments and Variables Affecting Course and Outcome. Berlin, Springer, 1988, pp 129-148.

Kessler RC, McGonagle KA, Zhao S, Nelson CB, Hughes $M$, Eshleman S, Wittchen H-U, Kendler KS: Lifetime and 12-month prevalence of DSM-III-R psychiatric disorders in the United States: Results from the National Comorbidity Survey. Arch Gen Psychiatry (in press).

Korff $\mathrm{M}$ von, Eaton WW: Epidemiologic findings on panic, in Baker $R$ (ed): Panic Disorder: Theory, Research and Therapy. New York, Wiley, 1989, pp 35-50.

Lemeshow S, Hosmer DW, Klar J, Lwanga SK (eds): Adequacy of Sample Size in Health Studies. Published on Behalf of the World Health Organization. New York, Wiley, 1990.

Margraf J, Ehlers A, Wittchen $\mathrm{H}-\mathrm{U}$ : Panic attacks in nonclinical subjects, in Hand I, Wittchen H-U (eds): Panic and Phobias. 2. Treatments and Variables Affecting Course and Outcome. Berlin, Springer, 1988, pp 103-117.

Margraf J, Schneider S (eds): Panik. Angstanfälle und ihre Behandlung. Berlin, Springer, 1989.

Wittchen H-U: Natural course and spontaneous remissions of untreated anxiety disorder: Results of the Munich Follow-up Study (MFS), in Hand I, Wittchen H-U (eds): Panic and Phobias. 2. Treatments and Variables Affecting Course and Outcome. Berlin, Springer, 1988, pp 3-17.

Wittchen H-U: Der Langzeitverlauf unbehandelter Angststörungen: Wie häufig sind Spontanremissionen? Verhaltenstherapie 1991;1: 273-282.

Wittchen H-U: Komorbidität bei Angststörungen - Häufigkeit, ätiologische und klinische Implikationen, in Kasper S, Möller H-J (eds): Diagnostik und Therapie von Angst/Panikerkrankungen. Gräfelfing, SM-Verlag, 1993, pp 60-69.

Wittchen H-U: Reliability and validity studies of the WHO-Composite International Diagnostic Interview (CIDI). A critical review. J Psychiatric Res (im Druck).

Wittchen H-U, Burke JD, Semler G, Pfister H, von Cranach M, Zaudig M: Recall and dating reliability of psychiatric symptoms test-retest reliability of time related symptom questions in a standardized psychiatric interview (CIDI/DIS). Arch Gen Psychiatry 1989;46:437-443.

Wittchen H-U, Essau CA: Comorbidity of anxiety disorders and depression: Does it affect course and outcome? J Psychiatry Psychobiol 1989;4:315-323.

Wittchen.H-U, Essau CA: The epidemiology of panic attacks, panic disorder and agoraphobia, in Walker JR, Norton R, Ross CA (eds): 
Panic Disorder and Agoraphobia. A Guide for the Practitioner. Pacific Grove, Brooks/Cole, 1991, pp 103-149.

Wittchen $\mathrm{H}-\mathrm{U}$, Essau CA: Epidemiology of panic disorder: Progress and unresolved issues. J Psychiatric Res (im Druck, 1993).

Wittchen H-U, Essau CA, Krieg JC: Anxiety disorders: Similarities and differences of comorbidity in treated and untreated groups. Br J Psychiatry 1991;159(suppl 12):23-33.

Wittchen H-U, Essau CA, Zerssen D von, Krieg CJ, Hecht H: Lifetime and six-month prevalence of mental disorders in the Munich Follow-up Study. Eur Arch Psychiatry Clin Neurosci 1992;241: 247-258.

Wittchen H-U, Rupp HU: Diagnostic Interview Schedule (DIS) Version II (deutsche Übersetzung). München, Max-Planck-Institut für Psychiatrie, 1981.
Wittchen H-U, Semler G: Composite International Diagnostic Interview (CIDI, version 1.0). Weinheim, Beltz. Test, 1990.

Wittchen H-U, Zerssen D von (Hrsg): Verläufe behandelter und unbehandelter Depressionen und Angststörungen. Eine klinischpsychiatrische und epidemiologische Verlaufsuntersuchung. Berlin, Springer, 1987.

Sonderdruckbestellungen an:

Prof. Dr. H.-U. Wittchen

Psychiatrische Klinik

Max-Planck-Institut für Psychiatrie

Kraepelinstraße 2

D-80804 München (BRD)

\section{Verhaltenstherapie und Systemtherapie}

\section{Begegnung - Unterschiede - Praxisdemonstrationen}

\section{Bad Dürkheim, 19. März 1994}

\section{Referenten:}

Schiepek - Koppenhöfer - Tröscher - Hüfner • Deissler • Kosarz ·Schwarz $\cdot$ Becht $\cdot$ Schöll $\cdot$ Lieb u.a.

Hauptthemen:

- Menschenbildannahmen von ST und VT und praktische Konsequenzen für die Therapie

- Was ist ein Symptom? - Was ist eine Veränderung? Antworten aus verhaltenstherapeutischer und systemtheoretischer Sicht

- Live Supervision: VT und ST supervidieren einen Fall (Fallvorstellung - VT-Supervision - ST-Supervision Kommentare)

- Seele und Technik in der Psychotherapie

- Konzept und Praxis einer verhaltenstherapeutischen Familientherapie

- VT und ST: Schulendialog und Psychotherapeutengesetz (Teilnehmer: Referenten des Tages) 